Euskal ikerketen aldizkaria | Revue d'études basques |

Revista de estudios vascos | Basque studies review

$5 \mid 2000$

Numéro $\mathrm{V}$

\title{
Les Basques dans l'Ouest américain (1900-1910)
}

Marie-Pierre Arrizabalaga

\section{OpenEdition}

Journals

Édition électronique

URL : http://journals.openedition.org/lapurdum/1340

DOI : $10.4000 /$ lapurdum. 1340

ISSN : 1965-0655

Éditeur

IKER

Édition imprimée

Date de publication : 1 octobre 2000

Pagination : 335-350

ISBN : 2-84127-161-7

ISSN : $1273-3830$

Référence électronique

Marie-Pierre Arrizabalaga, « Les Basques dans l'Ouest américain (1900-1910) », Lapurdum [En ligne],

5 | 2000, mis en ligne le 01 juin 2009, consulté le 01 juillet 2020. URL : http://journals.openedition.org/ lapurdum/1340 ; DOI : https://doi.org/10.4000/lapurdum.1340 


\section{Les Basques dans l'Ouest américain \\ (1900-1910')}

Marie-Pierre Arrizabalaga²

Université de Cergy-Pontoise

L'immigration massive des Basques dans l'Ouest américain a débuté pendant la première décennie du XX' siècle. Certes, certains s'y sont installés bien avant 1900 ; cependant, ils étaient peu nombreux et résidaient dans les régions côtières de la Californie, aux abords de San Diego et de Los Angeles. À partir de la fin du XIX siècle, l'Ouest des États-Unis a attiré de plus en plus d'immigrants de telle sorte qu'en 1900, 986 Basques français et espagnols ont été répertoriés dans le recensement. À cette époque, ils ne s'établissaient pas seulement sur les côtes californiennes, mais aussi à l'intérieur des terres, dans la Sierra Nevada, voire dans les États du Nevada et de l'Idaho. Ces régions isolées n'ont cessé ensuite d'attirer davantage d'immigrants car, selon le recensement de 1910, la population basque française et espagnole y était près de dix fois supérieure à celle de 1900 (soit 8398 individus contre 986 en 1900). Visiblement, l'Ouest américain connaissait une première vague d'immigration massive, tout particulièrement pendant les années allant de 1905 à 1909. Cette période ne s'était pas seulement caractérisée par une forte immigration, mais aussi et surtout par une forte expansion économique, à la fois agricole et industrielle, dont le développement dépendait de la présence d'une main d'œuvre masculine de plus en plus importante.

\section{Objectifs}

Le but du présent article consiste précisément à étudier cette immigration basque dans l'Ouest américain entre 1900 et 1910 et les comportements encore méconnus de ces 8398 individus résidant dans ces contrées isolées, notamment dans les régions autour de Los Angeles, San Francisco, Reno (Nevada) et Boise (Idaho). Il s'agit d'un phénomène qui n'a pas fait l'objet d'une étude globale approfondie car les archives américaines les concernant sont peu nombreuses et très incomplètes. En effet, les registres fonciers, qui ne concernaient que les propriétaires de biens, soumis à l'impôt, donnent une idée parcellaire de la population immigrante installée dans ces contrées à cette époque. En outre, les registres des services de l'immigration ne sont pas encore accessibles au public. Enfin, cette population, aujourd'hui décédée, a laissé peu de témoignages. Les seules archives 
exhaustives à notre disposition sont les listes nominatives par commune collectées lors des recensements de 1900 et 1910 dans les États de Californie, Nevada, Idaho et Wyoming, que nous avons dépouillées dans le cadre de notre analyse ${ }^{2}$.

De nombreuses études ont pour objectif de mieux comprendre l'immigration des Basques aux États-Unis. L'étude de référence dans le domaine et la plus exhaustive demeure celle de William A. Douglass et Jon Bilbao, Amerikanuak ${ }^{3}$, qui retrace l'histoire de l'émigration des Basques du nord (français) et du sud (espagnols) depuis la découverte de l'Amérique jusqu'à nos jours. Cette émigration a débuté en Argentine et en Uruguay dès le XVII siècle, s'est ensuite étendue dans toute l'Amérique du Sud au XVIII siècle, puis s'est dirigée vers le Mexique au XIX siècle et enfin, vers les États-Unis depuis le début de la ruée vers l'or jusqu'à nos jours. Il s'agit d'une étude globale sur les destins migratoires et professionnels des Basques français et espagnols en Amérique. Cet historique cependant ne comprend pas de statistiques spécifiques corroborant les modèles migratoires qu'ils ont ainsi élaborés. Nous tenterons dans notre analyse d'aller au-delà des conclusions de William A. Douglass et Jon Bilbao et par conséquent, de compléter cette recherche.

D'autres études sur les Basques en Amérique ont été entreprises, la plupart plus récentes que celle-ci. Pierre Lhande a étudié les expériences d'émigrants basques aux États-Unis entre la fin du XIX $X^{e}$ siècle et le milieu du XXe siècle ${ }^{4}$. Cependant, ces informations collectées lors de séjours prolongés en Amérique à cette époque ne sont guère exhaustives et relèvent plutôt de l'anecdote. Elles se composent principalement de témoignages d'expériences individuelles d'immigrants installés dans le nouveau continent, de simples récits sans support statistique. Enfin, la grande majorité des études concernent la période récente. Il s'agit d'études locales sur les colonies basques de San Francisco ${ }^{5}$, Stockton 6 , Bakersfield ${ }^{7}$ en Californie, Reno, Eliko dans le Nevada, et Boise en Idaho ${ }^{8}$, ou bien des études régionales sur les colonies de Californie, du Nevada et de l'Idaho'. Enfin, pour la période récente (post 1945), Eric Branaa a entrepris une étude sur l'émigration des Basques aux États-Unis à partir des archives de Charles Iriart, agent de l'émigration entre 1945 et $1972^{10}$. Dans un autre registre, soulignons les travaux de certains auteurs tels que Annick Foucrier ${ }^{11}$, Hernán Gustavo Otero ${ }^{12}$ et Jose C. Moya ${ }^{13}$ spécialistes de l'émigration espagnole ou française, des études qui, dans le cadre de cas régionaux particuliers présentent des exemples basques.

L'intérêt de la présente étude est de mieux comprendre l'immigration des Basques entre 1900 et 1910, car il s'agit de la première vague d'immigration massive aux Etats-Unis. Cependant, ce phénomène est encore peu connu et, pour la première fois, une analyse entreprend une comparaison systématique entre les comportements des Basques français et des Basques espagnols. Tandis que l'immigration basque française a débuté avant 1900 (comme Annick Foucrier l'a bien démontré dans ses travaux $\left.{ }^{14}\right)$, l'immigration basque espagnole était très récente. Notre étude globale et régionale tiendra compte à la fois de cette disparité nationale et régionale (le Pays Basque nord, français, et le Pays Basque sud, espagnol), des expériences individuelles et familiales telles qu'elles apparaissent dans les États de l'Ouest américain, et enfin, de leur destin dans les villes, les montagnes 
ou les déserts. L'analyse détaillée de leurs comportements migratoires, professionnels et matrimoniaux nous permettra de mieux cerner la nature de leurs destins migratoires. L'originalité de notre étude vient aussi de la nature des sources et de son analyse. Grâce au dépouillement systématique du recensement de 1910, que personne n'a encore exploité, nous déterminerons l'origine géographique des immigrants et étudierons leurs comportements. Nous constaterons que les Basques français, d'origine rurale, et les Basques espagnols, d'origine plus urbaine, ont adopté des comportements migratoires, professionnels et matrimoniaux parfois très différents. Nous entreprendrons une comparaison systématique de leurs destins dans le but de démontrer que, par rapport aux Basques espagnols, les Basques français s'établissaient davantage dans les régions rurales de Californie et du Nevada, exerçaient des professions majoritairement agricoles, et n'hésitaient pas à se marier et se naturaliser Américains, formant ainsi des groupes familiaux installés définitivement aux États-Unis.

\section{Méthodologie}

Notre analyse se fonde sur le dépouillement systématique des listes nominatives de 1910 dans toutes les communes des quatre États américains où la population basque était concentrée : Californie, Nevada, Idaho et Wyoming. Nous y avons localisé 8398 individus. Une attention toute particulière a été portée aux individus qui, dans la colonne « lieu de naissance », se déclaraient français ou espagnols. Si leur nom de famille avait une consonance basque, nous relevions tous les renseignements civils les concernant. En outre, nous nous sommes arrêtés sur les individus nés aux États-Unis dont les parents étaient nés en France ou en Espagne ${ }^{15}$. Lorsque leur nom de famille avait une consonance Basque, nous relevions toutes les informations les concernant. Ainsi, la cohorte se compose de Basques français, de Basques espagnols et d'Américains d'origine basque. Les données les concernant sont les suivantes : nom, prénom, sexe, âge, statut matrimonial, nombre d'années de mariage, nombre d'enfants nés et survivants, lieu de naissance et celui des parents, année de leur entrée aux États-Unis, statut résidentiel (étrangers, résidents permanents, naturalisés ou américains), profession, statut professionnel (employés ou employeurs), langue parlée et écrite, niveau d'alphabétisation (quelle que fût la langue parlée), et enfin, situation foncière (locataires ou propriétaires). Nous avons ensuite procédé à l'informatisation de tous ces éléments.

L'analyse des données ci-dessus va permettre de mieux comprendre les destins de ces Basques, leur lieu de résidence et leur processus d'intégration dans la société américaine. La nature de ces informations a parfois posé des problèmes. Les épouses d'immigrants par exemple n'indiquaient pas leur nom de jeune fille. Elles étaient américaines, françaises ou espagnoles. Il était parfois difficile de déterminer avec certitude si ces femmes étaient réellement d'origine basque. En outre, pour les mêmes raisons, nous n'avons probablement pas localisé un certain nombre de femmes qui ont épousé des Américains ou des Européens car leur nom de jeune fille n'apparaissait pas sur les listes et leur nom d'épouse n'avait aucune consonance basque. En dépit de ces omissions, peu d'individus ont échappé à notre ana- 
lyse car, comme nous le verrons, à cette époque, les femmes constituaient un groupe minoritaire et résidaient en famille, avec leurs frères, leur mari ou leur fiancé ${ }^{16}$.

À titre de comparaison, nous avons choisi d'utiliser les informations concernant les 986 individus nés au Pays Basque ou d'origine basque qu'Tban Bilbao et Chantal de Eguiluz ont relevées dans le recensement de 1900, mais qu'ils n'ont pas exploitées ${ }^{17}$. Nous avons informatisé l'ensemble des données afin d'entreprendre ensuite une analyse détaillée et comparative des destins migratoires, professionnels et matrimoniaux de tous les Basques français et espagnols résidant dans l'Ouest des États-Unis ainsi que leur processus d'intégration et d'assimilation entre la fin du $\mathrm{XIX}^{e}$ siècle et le début du XX $\mathrm{X}^{\mathrm{e}}$ siècle.

\section{Origine géographique}

L'analyse des données de 1900 ainsi récoltées a permis d'observer parmi les résidents la présence d'un grand nombre d'Américains d'origine basque, soit $40,1 \%$ d'individus nés aux États-Unis, de parents immigrés dans la seconde moitié du XIX $X^{e}$ siècle. Les autres membres de la cohorte se composent d'hommes et de quelques femmes qui ont immigré entre 1890 et 1900 pour la plupart, dont $30,3 \%$ étaient d'origine basque française et $29,6 \%$ d'origine basque espagnole (soit $59,9 \%$ d'individus au total) (voir Tableau 1). Les immigrants les plus anciens étaient donc d'origine française. Ils s'étaient installés dans ces contrés éloignées de l'Amérique depuis plusieurs décennies parfois et y avaient fondé une famille ${ }^{18}$. C'est pourquoi deux Basques sur cinq environ étaient nés aux États-Unis, leurs parents, d'origine française, ayant quitté le Pays Basque bien avant 1900. Dans un premier temps, certains d'entre eux ont peut-être émigré en Argentine, en Uruguay ou au Mexique, en suivant la chaîne d'immigration traditionnelle que William A. Douglass et Jon Bilbao ont clairement identifiée dans leur ouvrage ${ }^{19}$. Quoi qu'il en soit, les Basques français en 1900 étaient fortement implantés dans l'Ouest des États-Unis, notamment sur les terres intérieures du comté de Los Angeles, au sud de la Californie. Les nouveaux immigrants continuaient à entrer aux États-Unis par le port de Los Angeles, cependant ils arrivaient de plus en plus par le port de San Francisco en direction de la Sierra Nevada.

Tableau

Origine des Basques résidant dans l'Ouest des États-Unis en 1900 et 1910.

\begin{tabular}{|c|c|c|}
\hline Origines & $\mathbf{1 9 0 0}$ & $\mathbf{1 9 1 0}$ \\
\hline Basques français & $299(30,3 \%)$ & $2014(24,1 \%)$ \\
\hline Basques espagnols & $292(29,6 \%)$ & $3604(43,1 \%)$ \\
\hline Basques américains & $395(40,1 \%)$ & $2751(32,8 \%)$ \\
\hline Total & $986(100 \%)$ & $8369(100 \%)$ \\
\hline
\end{tabular}


L'analyse du recensement de 1910 met donc en évidence un phénomène encore plus remarquable : une augmentation rapide et récente du nombre de Basques résidant dans l'Ouest des États-Unis : soit 8398 individus, contre 986 en 1900. Parmi les individus de cette cohorte, on remarque que $67,2 \%$ sont originaires du Pays Basque, dont $24,1 \%$ sont du Pays Basque français et $43,1 \%$ du Pays Basque espagnol. Les autres (soit 32,8\%) sont nés et résident aux États-Unis avec leurs parents, soit $32,8 \%$ de la cohorte seulement (voir Tableau 1). Ces observations nous permettent ainsi de constater le rajeunissement de la cohorte, conséquence de l'arrivée récente et massive de jeunes immigrants et de la naissance de nombreux enfants d'anciens immigrants entre 1900 et 1910 . En outre, à la différence de 1900 où notre population se composait majoritairement de Basques français ou de descendants d'origine basque française, la cohorte de 1910 compte davantage de Basques espagnols. Ces derniers semblent attirés par les opportunités économiques offertes aux États-Unis à une époque où l' Argentine, l'Uruguay, voire le Mexique connaissaient des difficultés économiques croissantes. Leur arrivée était très récente et en nombre croissant à mesure que les années passaient. Peu nombreux à immigrer entre 1900 et 1903 (environ 200 par année), leur nombre n'a cessé d'augmenter en 1904 et en 1905 (jusqu'à 313 en 1905) et atteint des sommets particulièrement élevés entre 1906 et 1909. À l'image de l'immigration à l'échelle nationale, c'est en 1907 que le plus grand nombre d'entre eux a immigré ${ }^{20}$ (657 d'entre eux, voir Annexe A. 1.). En outre, cette immigration récente se composait de jeunes hommes célibataires, ce qui explique que la colonie non seulement rajeunissait, mais qu'en plus, elle se masculinisait. En effet, près des trois quarts étaient des hommes : $74,4 \%$ d'hommes et $25,6 \%$ de femmes en 1910 , contre $70,9 \%$ d'hommes et $29,1 \%$ de femmes en 1900 (voir Tableau 2). Cette immigration ayant débuté depuis quelques années seulement, elle n'attirait que les hommes. Les femmes suivaient le mouvement peu à peu.

Tableau 2

Sexe de la population basque des échantillons de 1900 et 1910.

\begin{tabular}{|c|c|c|}
\hline Sexe & $\mathbf{1 9 0 0}$ & $\mathbf{1 9 1 0}$ \\
\hline Hommes & $488(70,9 \%)$ & $4511(74,4 \%)$ \\
\hline Femmes & $200(29,1 \%)$ & $1553(25,6 \%)$ \\
\hline Total & $688(100 \%)$ & $6064(100 \%)$ \\
\hline
\end{tabular}

Destins géographiques

L'installation des immigrants a non seulement évolué dans le temps, mais aussi dans l'espace. En effet, en 1900, 75,6\% d'entre eux résidaient en Californie, contre $18,3 \%$ dans le Nevada et $6,2 \%$ en Idaho (voir Tableau 3). À cette époque, aucun n'avait encore atteint le Wyoming. Ils ne vivaient pas forcément éparpillés, mais plutôt dans des comtés spécifiques et dans les villes : notamment autour de Los Angeles (28,3 \%), San Francisco (13,1 \%), entre Reno et Elko dans le nord du Nevada (16,2\%) et autour de Boise en Idaho (6,2\%). Les autres résidaient à l'in- 
térieur des terres, dans la Sierra Nevada, entre les comtés de Bakersfield au Sud à celui de Chico au Nord de l'État de Californie, ou dans les montagnes et le désert du Nevada. Les individus résidant à Los Angeles en 1900 étaient principalement issus d'une immigration ancienne qui comptait beaucoup de Basques français ou d'Américains d'origine basque française. Ils accueillaient une population immigrante pour la plupart originaire du Pays Basque français. Une fois débarqués à Los Angeles, ils restaient dans la ville ou rejoignaient les régions d'élevage intensif du sud de la Californie, ceux de la Sierra Nevada et du nord du Nevada. Par contraste, les Basques espagnols, immigrés depuis peu, arrivaient par le port de San Francisco où ils s'installaient en grand nombre ou bien s'en éloignaient pour s'implanter dans les comtés du nord de la Sierra du Nevada, puis ceux du nord du Nevada et enfin et surtout, autour de Boise, en Idaho. Ainsi, à cette époque, deux chaînes d'immigration évoluaient en même temps : celle, composée de Basques français, qui démarrait à Los Angeles, longeait la Sierra Nevada, vers le nord du Nevada, puis celle, composée de Basques espagnols, qui démarrait à San Francisco, en direction du nord du Nevada, vers Boise, en Idaho. D'origine française ou espagnole, beaucoup décidaient de s'établir dans les villes, notamment Los Angeles, San Francisco, Reno et Boise. Contrairement à ce qu'a longtemps affirmé l'historiographie, les immigrants ne fuyaient pas les villes ${ }^{2} 1$. En 1900, $41,4 \%$ des immigrants de la cohorte vivaient dans les villes de Los Angeles et San Francisco principalement. Dès le début du siècle, ils n'hésitaient pas à se réunir dans les hôtels basques, devenus pour l'occasion des centres culturels, des lieux de réunion et de recrutement dans les principales villes des quatre États et tout particulièrement Los Angeles et San Francisco, les deux ports d'entrée les plus fréquentés dans l'Ouest des États-Unis à cette époque ${ }^{22}$.

Tableau 3

Répartition spatiale de la population basque de 1900.

\begin{tabular}{|c|c|c|}
\hline État & 1900 & 1910 \\
\hline Californie & $745(75,6 \%)$ & $6267(74,6 \%)$ \\
\hline Nevada & $180(18,3 \%)$ & $971(11,6 \%)$ \\
\hline Idaho & $61(6,2 \%)$ & $999(11,9 \%)$ \\
\hline Wyoming & $0(0 \%)$ & $161(1,9 \%)$ \\
\hline Total & $986(100 \%)$ & $8398(100 \%)$ \\
\hline
\end{tabular}

La situation évolua quelque peu au cours de la première décennie du $\mathrm{XX}^{\circledR}$ siècle. Même si les trois quarts des immigrants s'établissaient en Californie plutôt que dans les trois autres États, ils étaient nombreux à résider dans le Nevada, l'Idaho et à présent, le Wyoming (voir Tableau 3). Ils étaient non seulement beaucoup plus nombreux à immigrer, mais ils étaient pour la plupart originaires du Pays Basque espagnol. Tandis que la Californie restait une région de prédilection pour beaucoup de Basques français ou espagnols, ces derniers s'établissaient près de San 
Francisco et autour de Boise plutôt que dans le nord du Nevada ou le Wyoming. Les Basques français eux s'installaient en Californie (Los Angeles et la Sierra Nevada), dans le nord du Nevada et dans le Wyoming. Cette population se sédentarisait davantage en Californie qu'ailleurs car de nombreuses femmes et de familles y résidaient. En effet, en Californie, les hommes étaient deux fois plus nombreux que les femmes alors que dans le Nevada, ils étaient cinq fois plus nombreux, six fois et demi plus nombreux dans l'Idaho, et sept fois et demi plus nombreux dans le Wyoming (voir Annexe A.2.). À mesure que l'on s'éloigne de Los Angeles et de San Francisco, la colonie se composait principalement d'hommes, les conditions de vie (le climat difficile et l'isolement) décourageant probablement beaucoup de femmes à les suivre. Non seulement la cohorte se masculinisait, mais elle se ruralisait, la proportion d'individus vivant en milieu rural augmentant plus rapidement que celle des villes.

Ces observations nous mènent à plusieurs conclusions. Entre 1900 et 1910, les Basques espagnols arrivaient en grand nombre du Pays Basque par San Francisco, le port d'entrée de la nouvelle immigration et le lieu de résidence de beaucoup d'entre eux (de 129 en 1900 à 1416 en 1910). Dès lors, la ville de Los Angeles ne constituait plus la première ville d'accueil et le premier maillon de la chaîne migratoire des Basques dans l'Ouest américain. San Francisco a pris le relais, conséquence de son expansion économique récente. Les jeunes immigrants, notamment les Basques espagnols, choisissaient cette destination, attirés par les opportunités industrielles et commerciales de la ville dans la première décennie du XX $\mathrm{XX}^{e}$ siècle. Dès lors, San Francisco constituait le premier maillon de cette nouvelle chaîne migratoire qui se poursuivait ensuite vers le nord du Nevada et atteignait l'Idaho et le Wyoming. Ainsi, les immigrants optaient pour des destins migratoires différents les uns des autres. Les Basques français étaient attirés tout particulièrement par la Californie et le Wyoming, et dans une moindre mesure par le Nevada, tandis qu'ils se désintéressaient totalement de l'Idaho. En revanche, presque toute la colonie de San Francisco et celle de la région de Boise en Idaho se composait de Basques espagnols, de même que les deux tiers de la colonie du Nevada et la moitié de la colonie du Wyoming. Ainsi, les chaînes migratoires de ces immigrant: pendant la première décennie du xx siècle différaient selon leurs origines. Cela ne tenait pas à la langue, car tous parlaient le basque, mais plutôt à leurs origines géographiques, rurales pour les Basques français et urbaines pour les Basques espagnols. Ces différences apparaîtront aussi lors de notre analyse portant sur les destins professionnels.

\section{Destins professionnels}

Nous avons vu que dans la première décennie du $\mathrm{XX}^{e}$ siècle, la colonie basque se masculinisait et se ruralisait. En dépit de cette observation, de moins en moins d'individus exerçaient des professions agricoles comme éleveurs ovins et bovins. En effet, 42,9\% des individus seulement se déclaraient éleveurs en 1910 contre $66,4 \%$ au recensement précédent (voir Annexe A.3.). Tandis que la plupart des individus vivaient de revenus agricoles en 1900, 33,6\% seulement exerçaient des 
professions non-agricoles comme ouvriers, qualifiés ou non qualifiés, comme employés de commerce (dans les laveries et les boulangeries), employés de services (dans l'hôtellerie notamment) ou dans les professions libérales. Ensuite, la situation s'est reversée. Même en milieu rural (où résidaient 70,8 \% des individus), les immigrants exerçaient des professions non-agricoles comme ouvriers qualifiés ou non-qualifiés et comme employés de commerce ou des services (soit $66,4 \%$ ), les professions libérales ou commerciales attirant encore peu d'immigrants $(3,8 \%$ seulement $)$.

Par ailleurs, les données nominatives de 1900 et 1910 indiquent que les Basques français étaient plus attachés à la vie rurale, et par conséquent, exerçaient davantage des professions agricoles que les Basques espagnols. Cela s'explique par la présence d'un plus grand nombre de Basques espagnols en ville, notamment à San Francisco et à Boise, en Idaho, où ils exerçaient des activités professionnelles diversifiées (comme ouvriers artisans, voire comme mineurs). En revanche, les Basques français (ceux résidant dans le sud de la Californie, dans le Wyoming et dans une moindre mesure, dans le nord du Nevada) limitaient leurs activités économiques et professionnelles à celles rattachées au secteur agricole ${ }^{23}$. Notons par exemple que $58,9 \%$ de la colonie d'Idaho exerçaient des professions agricoles, les autres $(33,7 \%)$ préférant pour des emplois non-qualifiés à Boise, la capitale de l'État. Dans les États du Nevada et du Wyoming où beaucoup de Basques français ont choisi de vivre, les emplois agricoles y étaient majoritaires ${ }^{24}$.

Comment expliquer ce phénomène? Nous pourrions certes croire que, parce qu'ils se sédentarisaient en ville, les Basques espagnols avaient tendance à s'intégrer plus rapidement à la vie et à la culture américaine que les Basques français. Cependant, William A. Douglass et Jon Bilbao ont interprété ce phénomène d'une autre manière, justifiant les différences de comportements des immigrants par leurs origines géographiques et les modes de vie économiques et professionnels dans leur pays ${ }^{25}$. En effet, selon les deux auteurs, les Basques espagnols avaient des comportements différents des Basques français parce que les premiers étaient originaires de villes et villages industriels ou miniers et les derniers de villages ruraux. Ainsi, nous pourrions interpréter les données des recensements de la manière suivante. Tandis que les Basques français, originaires de villages ruraux agricoles, s'efforçaient de perpétuer leurs pratiques familiales en exerçant des professions agricoles dans la Sierra Nevada et le nord du Nevada, les Basques espagnols, originaires des villes portuaires et minières de la province de Vizcaya (entre les villes de Bilbao et San Sebastian) pour la plupart, se destinaient à des emplois qualifiés ou non qualifiés dans les villes américaines. Ces différences peuvent certes s'expliquer à partir du fait que les Basques français et les Basques espagnols avaient des origines géographiques et des modes de vie différents, mais elles sont aussi le reflet d'histoires politiques et de passé idéologique différents ${ }^{26}$. Comme nous le verrons, leur rattachement à la France ou à l'Espagne explique certaines différences dans les comportements. Cependant, les données recensées ne peuvent corroborer un lien entre comportements individuels et expériences politiques. Ce qui est certain, c'est que leurs origines françaises ou espagnoles expliquent l'existence de chaînes migratoires différentes, voire de réseaux migratoires différents, 
qui inévitablement affectaient les comportements des uns et des autres, canalisaient leur destins professionnels et expliquent leur degré d'intégration plus ou moins élevé.

\section{Processus d'intégration}

Nous pensons que l'analyse des destins matrimoniaux des immigrants peut nous aider à déterminer leur degré d'intégration aux États-Unis. En ce qui concerne la population adulte de notre échantillon, nous observons une nombre important de célibataires $(56,2 \%$ en 1900 et $54,7 \%$ en 1910). Cela n'est pas surprenant compte tenu du fait que la majorité des individus étaient des jeunes hommes résidant aux États-Unis depuis peu. Tandis que la proportion de célibataires du sexe masculin restait élevée pendant toute la période, les deux tiers des femmes étaient mariées en 1900 et 1910. Une des raisons pour lesquelles les femmes mariées étaient aussi nombreuses provient du déséquilibre numérique des femmes par rapport aux hommes. Cela ne veut pas pour autant dire qu'elles souhaitaient s'intégrer plus rapidement que les hommes. C'était probablement une question de nécessité, voire de survie pour elles car les emplois féminins étaient peu nombreux et probablement peu rémunérés, notamment dans les comtés isolés. En outre, du fait du déséquilibre numérique entre hommes et femmes, le marché matrimonial était avantageux pour les femmes, leur assurant la stabilité économique qu'elles ne pouvaient acquérir seules. Enfin, certaines femmes suivaient ou rejoignaient leur mari ou leur fiancé déjà installé dans le nouveau continent. Toutes ces raisons expliquent pourquoi proportionnellement plus de femmes que d'hommes étaient mariées $(56,9 \%$ en 1900 et $69,9 \%$ en 1910 contre respectivement $32 \%$ et $31,6 \%$ d'hommes). Par ailleurs, $33 \%$ des hommes en 1900 et $24,2 \%$ des hommes en 1910 ont émigré seuls, laissant leur épouse au Pays. Ce constat cependant ne nous permet pas d'affirmer que les femmes s'intégraient plus rapidement que les hommes (voir Annexe A.4.).

Tableau 3

Lieu du mariage des Basques selon les recensements de 1900 et 1910.

\begin{tabular}{|c|c|c|}
\hline Lieu de mariage & $\mathbf{1 9 0 0}$ & $\mathbf{1 9 1 0}$ \\
\hline France & $18(6,7 \%)$ & $103(4,0 \%)$ \\
\hline Espagne & $6(2,2 \%)$ & $297(11,4 \%)$ \\
\hline Amérique avec un Basque & $126(46,6 \%)$ & $1183(45,4 \%)$ \\
\hline Amérique avec un non-Basque & $22(8,2 \%)$ & $246(9,4 \%)$ \\
\hline Conjoint absent & $89(33,0 \%)$ & $632(24,2 \%)$ \\
\hline Inconnu & $9(3,3 \%)$ & $147(5,6 \%)$ \\
\hline Total & $270(100 \%)$ & $2608(100 \%)$ \\
\hline
\end{tabular}


Il semble que les jeunes immigrants, hommes ou femmes, ne souhaitaient pas s'intégrer rapidement à la société américaine. En réalité, le processus d'intégration évoluait de manière progressive d'une génération à l'autre. Ils s'efforçaient de préserver des pratiques familiales traditionnelles et de reconstituer des groupes familiaux solides avec d'autres Basques. Ils n'hésitaient pas à se marier, cependant leurs pratiques matrimoniales demeuraient culturellement extrêmement endogames. En effet, $8,9 \%$ des Basques en 1900 et 15,4\% en 1910 se sont mariés au Pays Basque et vivaient aux États-Unis avec leur épouse. Par ailleurs, les immigrants célibataires, hommes et femmes, n'épousaient pas des Américains, mais plutôt des immigrants basques ou des Américains d'origine basque. Ainsi, 46,6\% des individus mariés aux États-Unis avant 1900 et 45,4\% de ceux mariés aux ÉtatsUnis avant 1910 ont épousé des Basques ou des Américains d'origine basque. Enfin, 8,2\% des immigrants en 1900 et 9,4\% en 1910 seulement ont accepté des alliances matrimoniales exogames, en épousant des hommes ou des femmes de cultures différentes de la leur (voir tableau 4).

Certes, la plupart des immigrants ne souhaitaient pas s'intégrer immédiatement à la culture américaine et élaboraient des pratiques matrimoniales extrêmement endogames. Cependant, ils n'hésitaient pas à s'installer définitivement aux ÉtatsUnis. Leur sédentarisation et la reconstitution d'un noyau culturel et familial traditionnel étaient en fait des étapes intermédiaire dans leur processus d'intégration. En fait, il faut différencier le processus d'intégration des Basques français par rapport à celui des Basques espagnols. Les premiers se mariaient en plus grand nombre et étaient davantage intégrés que les seconds. En outre, ils étaient pour la plupart propriétaires et résidents permanents. Cela s'explique probablement par le fait que leur installation dans l'Ouest des États-Unis était bien pius ancienne et que leur implantation était par conséquent plus grande. Par le mariage (même endogame) et l'achat de biens fonciers (terres et maisons), ils pérennisaient leur projet migratoire. Par contraste, plus des trois quarts $(77,2 \%)$ des immigrants basques espagnols de Boise, arrivés en Idaho entre 1905 et 1910 (soit 999 individus en 1910) étaient célibataires (principalement des hommes). Parmi ceux qui étaient dans leur province d'origine le tiers n'était pas accompagné des épouses. Tandis que les uns, très nombreux, refusaient de se marier, les autres attendaient l'arrivée prochaine de leurs femmes ou espéraient entrer chez eux après quelques années de séjour aux Etats-Unis. En dépit de leur implantation urbaine, la colonie de Boise semblait moins intégrée à la vie et la culture américaine que les Basques français.

Il en ressort que la plupart des immigrants, notamment ceux d'origine française, épousaient des compatriotes ou, cas plus rares, des Américains d'origine basque. Ainsi, en 1910, ils restaient attachés à leurs racines et à leur culture. Ils protégeaient ainsi leurs pratiques culturelles en acceptant des alliances matrimoniales endogames à la fois en milieu urbain et en milieu rural. Ceux qui par contraste contractaient des alliances matrimoniales exogames (avec des individus de cultures différentes) étaient souvent des Américains nés aux États-Unis mais d'origine basque qui exploitaient toutes les ressources humaines et culturelles du nouveau continent, des Américains qui étaient entièrement assimilés à la culture américaine. Rares étaient les immigrants basques (de la première génération) qui 
épousaient des Américains non-basques car cela impliquait un processus d'intégration trop rapide.

\section{Processus $d$ 'assimilation}

Peu d'immigrants à cette époque s'assimilaient à la culture du nouveau monde au point de prendre la citoyenneté américaine. Leur processus d'assimilation était lent. Certes, deux cinquièmes de la cohorte en 1900 (soit 40,1\%) et un tiers de celle de 1910 (soit 33,1\%) étaient nés aux États-Unis. Ils étaient donc américains. Il s'agissait des enfants de Basques français arrivés à San Francisco et au sud de la Californie depuis de nombreuses années ou de Basques espagnols de la région de San Francisco. Ils s'y étaient sédentarisés et y avaient fondé une famille. Le processus d'assimilation semblait évoluer très progressivement. En fait, il était en meilleure voie en 1900 qu'en 1910. Tandis que les premiers vivaient en Amérique depuis longtemps et avaient reconstitué un noyau familial solide dans quelques villes ou comtés californiens, les seconds, plus jeunes et arrivés très nombreux depuis peu, vivaient en quasi autarcie, notamment dans la ville de Boise ou dans certaines régions isolées, en montagne ou dans le désert, et étaient par conséquent peu assimilés. Cela explique pourquoi en 1900,25,3\% des immigrants avaient pris la nationalité américaine et seulement 14,3\% en 1910 (voir Tableau 5). Notons en outre que $6,8 \%$ des immigrants de la cohorte de 1910 étaient des résidents permanents. Par contraste, 74,7\% des individus en 1900 et $78,9 \%$ en 1910 conservaient leur statut d'étrangers.

Tableau 5

Statut résidentiel des immigrants en 1900 et 1910.

\begin{tabular}{|c|c|c|}
\hline Statut résidentiel & $\mathbf{1 9 0 0}$ & $\mathbf{1 9 1 0}$ \\
\hline Étrangers & $293(74,7 \%)$ & $3062(78,9 \%)$ \\
\hline Résidents permanents & 0 & $264(6,8 \%)$ \\
\hline Naturalisés & $99(25,3 \%)$ & $553(14,3 \%)$ \\
\hline Total & $392(100 \%)$ & $3879(100 \%)$ \\
\hline
\end{tabular}

Ainsi, les immigrants basques français étaient davantage assimilés à la société américaine en 1900 et en 1910 que les immigrants basques espagnols. Ces différences tenaient à la localisation géographique et à l'ancienneté de cette colonie. Ils n'hésitaient pas à se marier avec des Basques français, voire avec des américains d'origine basque française ${ }^{27}$. En réalité, plus d'un immigrant basque français sur quatre en 1910 (soit 26,8\%) s'était naturalisé américain et près d'un sur dix était devenu résident permanent. Globalement, plus d'un immigrant basque français sur trois en 1900 et 1910 était établi aux États-Unis de manière définitive, la plupart mariés à des Basques français ou à des Américains d'origine basque française (voir Tableau 6). L'assimilation des Basques français était perceptible aussi 
dans leur comportement vis-à-vis de la propriété. Ils étaient nombreux à être pro-priétaires de leur maison et de leurs terres. Plus individualistes et fidèles à la culture traditionnelle familiale, ils reconstituaient une cellule familiale traditionnelle autour du foyer.

\section{Tableau 6}

Statut résidentiel des immigrants Basquues français et des Basques espagnols.

\begin{tabular}{|c|c|c|c|c|}
\hline \multirow{2}{*}{$\begin{array}{c}\text { Statut résidentiel } \\
\text { Basques d'origine }\end{array}$} & \multicolumn{2}{|c|}{1900} & \multicolumn{2}{c|}{1910} \\
\hline Française & Espagnole & Française & Espagnole \\
\hline Etrangers & $10663,9 \%)$ & $187(82,7 \%)$ & $820(64,0 \%)$ & $3062(78,9 \%)$ \\
\hline Résidents permanents & 0 & 0 & $118(9,2 \%)$ & $264(6,8 \%)$ \\
\hline Naturalisés & $60(36,1 \%)$ & $99(25,3 \%)$ & $344(26,8 \%$ & $223(14,3 \%)$ \\
\hline Total & $166(100 \%)$ & $392(100 \%)$ & $1282(100 \%)$ & $3879(100 \%)$ \\
\hline
\end{tabular}

Le processus d'assimilation des immigrants basques espagnols est loin d'avoir atteint un tel niveau. En effet, en 1900, 82,9\% d'entre eux se déclaraient étrangers et $17,3 \%$ d'entre eux seulement étaient naturalisés (voir Tableau 6). La situation a peu évolué au cours de la première décennie du $x^{e}$ siècle. Bien que le nombre d'immigrants basques espagnols intégrés à la société américaine ait considérablement augmenté entre 1900 et 1910 , proportionnellement, $86,3 \%$ d'entre eux se déclaraient étrangers. Ils n'étaient que $8,1 \%$ à accepter la naturalisation américaine et $5,6 \%$ à choisir le statut de résidents permanents (voir Tableau 6). La grande majorité de ces individus, arrivés entre 1905 et 1909, n'envisageaient pas alors de passer leur vie aux États-Unis, et encore moins de renoncer à leur nationalité. Leur processus d'assimilation était beaucoup moins avancé que celui des immigrants basques français, d'autant que très peu d'entre eux étaient propriétaires de leur maison et leur terres. Cela ne veut pas dire pour autant qu'ils refusaient d'investir leur avenir dans un tel projet. Il était probablement trop tôt pour, légalement et sur une base volontaire, faire ce choix.

Les observations que l'on peut faire sur la pratique de la langue anglaise parmi les immigrants de la cohorte corroborent les conclusions ci-dessus. Certes, de plus en plus de Basques (Américains ou immigrants basques français ou basques espagnols) affirmaient parler l'anglais couramment en 1900 et en 1910. Cependant, proportionnellement, beaucoup plus de Basques espagnols (soit 61,8\%) que de Basques français (soit $31,3 \%$ ) déclaraient ne pratiquer que leur langue maternelle : le basque ou l'espagnol. Ainsi, près des deux tiers des Basques espagnols ne pouvaient s'exprimer en anglais, contre moins d'un tiers des Basques français.

L'analyse ci-dessus a clairement mis en évidence que les expériences migratoires des immigrants basques français contrastaient quelque peu avec celles des immigrants basques espagnols. C'est probablement pour cette raison que, dans le cadre des alliances matrimoniales, ils se mélangeaient peu. En effet, à partir de l'analyse des lieux de naissance des couples, nous constatons que peu d'entre eux 
ont contracté des alliances mixtes : entre un Basque français et un Basque espagnol. En effet, parmi les 1731 individus nés aux États-Unis, 47,4\% (soit 820 d'entre eux) sont issus d'unions strictement basques françaises (les deux parents d'origine basque française) et 40,4\% (soit 699 d'entre eux) sont issus d'unions strictement basques espagnoles (les deux parents d'origine basque espagnole). En revanche, seulement $12,2 \%$ de ces individus (soit $212 \mathrm{~d}$ 'entre eux) sont issus d'unions mixtes (un parent basque français et l'autre basque espagnol). Ainsi, non seulement les Basques français et les Basques espagnols empruntaient des chaînes migratoires quelque peu différentes et avaient des origines géographiques et politiques différentes, mais en plus, ils ne s'intégraient pas de la même manière à la société américaine et se côtoyaient peu. Au début du siècle, l'immigrant non seulement hésitait à se marier, mais, s'il se mariait, c'était avec un Basque ou, cas plus rare, un Américain d'origine basque dans le but de préserver des liens étroits avec leur pays. Il était bien intégré dans à la vie économique et culturelle américaine à partir du moment où il était marié, parlait la langue anglaise, était propriétaire de biens fonciers, et se naturalisait américain. Ce processus néanmoins était lent et concernait les immigrants les plus anciens, notamment les Basques français.

\section{Conclusion}

L'analyse des recensements étasuniens de 1900 et de 1910 a donc permis de mettre en évidence les modalités des destins migratoires des Basques aux ÉtatsUnis à la fin du XIX siècle et au début du XX $x^{e}$ siècle. Cette étude a révélé plusieurs éléments : la présence de Basques dans cette région bien avant 1900, surtout des Basques français établis en famille depuis de nombreuses années, notamment dans le sud de la Californie, dans la région de Los Angeles d'où ils étaient arrivés. Au cours de la première décennie, le nombre d'immigrants a considérablement augmenté, notamment entre 1906 et 1910. Il s'agissait de jeunes hommes, célibataires, pour la plupart d'origine basque espagnole qui arrivaient par le port de San Francisco. Ainsi, l'immigration basque dans l'Ouest des États-Unis a considérablement évolué en nombre et en nature. Tandis qu'en 1900, elle était peu importante (986 individus), majoritairement basque française et arrivée par Los Angeles, en 1910, elle était près de dix fois plus importante (8398 individus), majoritairement basque espagnole et arrivée par San Francisco. Ce changement est révélateur d'un phénomène remarquable. Non seulement la Californie, terre de prédilection pour tous les immigrants, attirait de plus en plus de Basques espagnols, mais leurs modalités d'intégration différaient quelque peu de celles des Basques français. En effet, ils n'empruntaient pas la même chaîne migratoire, optaient pour des destins professionnels et matrimoniaux parfois différents, et s'intégraient plus ou moins rapidement à la culture américaine.

Tandis que les Basques français s'établissaient davantage autour de Los Angeles, dans la Sierra Nevada, le nord du Nevada et à partir de 1900, dans le Wyoming, les Basques espagnols, eux, s'installaient dans les comtés autour de San Francisco, dans le nord du Nevada, et tout particulièrement dans l'Idaho. De la même manière, les uns et les autres avaient des destins professionnels bien diffé- 
rents. Tandis que les Basques français d'origine rurale et attachés aux valeurs traditionnelles de la terre, préféraient les professions liées au secteur agricole, parfois même lorsqu'ils vivaient en ville, les Basques espagnols, d'origine urbaine et industrielle, choisissaient des professions beaucoup plus diversifiées en ville comme en milieu rural. Inévitablement, ces origines influaient sur leurs comportements.

Cen'est pas parce que les Basques espagnols choisissaient des professions plus diversifiées qu'ils s'intégraient mieux à la culture américaine. D'autres indices sont révélateurs de leur degré d'intégration par rapport aux Basques français. D'une part, les Basques espagnols étaient de jeunes immigrants, célibataires et arrivés depuis peu aux États-Unis. D'autre part, ils étaient proportionnellement beaucoup moins nombreux à se marier. Lorsqu'ils se mariaient, ils préconisaient des alliances matrimoniales endogames, entre immigrants Basques espagnols ou entre Basques espagnols et américains d'origine basque espagnole. Leur intégration à la société américaine était loin d'avoir atteint celle des Basques français.

Les Basques français, eux, n'hésitaient pas à se marier et à élever une famille, notamment en Californie. Ils étaient davantage intégrés à la société américaine. Par le mariage, ils pérennisaient leur séjour aux États-Unis. Ils n'hésitaient pas ensuite à acheter des terres et à choisir les États-Unis comme leur lieu de résidence permanente, voire leur pays de naturalisation. Ainsi, la colonie basque française, plus ancienne, confortablement établie et composée d'immigrants et de leurs enfants américains, était davantage assimilée à la culture américaine. En outre, à la différence des Basques espagnols, beaucoup parlaient et écrivaient la langue anglaise. S'agissait-il d'une assimilation totale ? En dépit de leur volonté d'intégration, les Basques français gardaient de fortes attaches au Pays Basque et préservaient certaines de leurs pratiques familiales et culturelles. Leurs enfants, qui ont grandi aux États-Unis, épousaient parfois des immigrants Basques français, voire des Américains d'origine basque française. Ils préconisaient donc des alliances matrimoniales endogames non seulement pour eux, mais aussi pour leurs enfants.

L'analyse des alliances matrimoniales a permis en outre de constater que les mariages entre Basques espagnols et Basques français étaient peu courants. Cela tenait peut-être à leurs différences individuelles, voire culturelles et politiques. Les Basques français avaient des parcours migratoires et des destins professionnels et matrimoniaux quelque peu différents des Basques espagnols. Le degré d'intégration à la société américaine des premiers était peut-être trop avancé pour qu'ils côtoient les seconds. Par ailleurs, les mariages exogames étaient extrêmement rares à l'époque. C'est pourquoi seuls les descendants de la seconde génération en contractaient. L'immigrant basque français ou espagnol n'épousait presque jamais des individus de culture étrangère. Une analyse détaillée du recensement de 1920 permettrait de savoir de quelle manière dans la durée, les Basques français et espagnols arrivés entre 1900 et 1910 se sont intégrés à la société américaine. 


\section{Annexe}

Tableau A.1 Date d'arrivée des Basques selon le recensement de 1910

\begin{tabular}{|c|c|}
\hline Année d'Arrivée & Nombre d'immigrants \\
\hline 1900 & 208 \\
\hline 1901 & 162 \\
\hline 1902 & 196 \\
\hline 1903 & 196 \\
\hline 1904 & 304 \\
\hline 1905 & 313 \\
\hline 1906 & 406 \\
\hline 1907 & 657 \\
\hline 1908 & 536 \\
\hline 1909 & 465 \\
\hline
\end{tabular}

Tableau A.2. Sexe de la population adultes basques en 1910

\begin{tabular}{|c|c|c|c|}
\hline États - Sexe & Hommes & Femmes & Total \\
\hline Californie & $3005(68,8 \%)$ & $1363(31,2 \%)$ & 4368 \\
\hline Nevada & $812(83,6 \%)$ & $159(16,4 \%)$ & 971 \\
\hline Idaho & $866(86,7 \%)$ & $133(13,3 \%)$ & 999 \\
\hline Wyoming & $142(88,2 \%)$ & $19(11,8 \%)$ & 161 \\
\hline Total & $4825(74,2 \%)$ & $1674(25,8 \%)$ & 6499 \\
\hline
\end{tabular}

Tableau A.3. Professions des Basques en 1900 et 1910

\begin{tabular}{|c|c|c|}
\hline Professions & $\mathbf{1 9 0 0 ( \% )}$ & $\mathbf{1 9 1 0 ( \% )}$ \\
\hline Agriculture & $320(66,4 \%)$ & $2037(42,9 \%)$ \\
\hline Hôtellerie & $32(6,6 \%)$ & $329(6,9 \%)$ \\
\hline Mines & $5 \S 1,0 \%)$ & $380(8,0 \%)$ \\
\hline Blanchisseries & $16(3,3 \%)$ & $269(5,7 \%)$ \\
\hline Boulangeries & $2(0,4 \%)$ & $55(1,2 \%)$ \\
\hline Ouviiers non spécialisés & $54(11,2 \%)$ & $971(20,4 \%)$ \\
\hline Ouvriers spécialisés & $11(2,3 \%)$ & $194(4,1 \%)$ \\
\hline
\end{tabular}




\begin{tabular}{|c|c|c|}
\hline Professions & $1900(\%)$ & $1910(\%)$ \\
\hline Ouvriers des manufactures & $6(1,9 \%)$ & $28(0,6 \%)$ \\
\hline Ouvriers d'usine & 0 & $56(1,2 \%)$ \\
\hline Employés des services & $16(3,3 \%)$ & $114(2,4 \%)$ \\
\hline Employés des Chemins de fer & 0 & $66(1,4 \%)$ \\
\hline Pêcheurs & $1(0,2 \%)$ & $8(0,2 \%)$ \\
\hline Employés de bureau & 0 & $49(1,0 \%)$ \\
\hline Fonctionnaires & $1(0,2 \%)$ & $16(0,3 \%)$ \\
\hline Vente & 0 & $56(1,2 \%)$ \\
\hline Commerce & $6(1,2 \%)$ & $55(1,2 \%)$ \\
\hline Professions libérales & $9(1,9 \%)$ & $66(1,4 \%)$ \\
\hline Total & $482(100 \%)$ & $4749(100 \%)$ \\
\hline
\end{tabular}

Tableau A.4. Statut matrimonial des Basques adultes en 1900 et 1910

\begin{tabular}{|c|c|c|c|c|c|c|}
\hline $\begin{array}{c}\text { Statut } \\
\text { Matrimonial }\end{array}$ & Hommes & $\begin{array}{c}\mathbf{1 9 0 0} \\
\text { Femmes }\end{array}$ & Total & Hommes & $\begin{array}{c}1910 \\
\text { Femmes }\end{array}$ & Total \\
\hline Célibataires & $\begin{array}{c}321 \\
(46,9 \%)\end{array}$ & $\begin{array}{c}64 \\
(9,3 \%)\end{array}$ & $\begin{array}{c}395 \\
(56,2 \%)\end{array}$ & $\begin{array}{c}2960 \\
(49,1 \%)\end{array}$ & $\begin{array}{c}341 \\
(5,6 \%)\end{array}$ & $\begin{array}{c}3301 \\
(54,7 \%)\end{array}$ \\
\hline Mariés & $\begin{array}{c}156 \\
(22,8 \%)\end{array}$ & $\begin{array}{c}112 \\
(16,3 \%)\end{array}$ & $\begin{array}{c}268 \\
(39,1 \%)\end{array}$ & $\begin{array}{c}1417 \\
(23,5 \%)\end{array}$ & $\begin{array}{c}1085 \\
(18,0 \%)\end{array}$ & $\begin{array}{c}2502 \\
(41,5 \%)\end{array}$ \\
\hline Veufs & 10 & $\begin{array}{c}19 \\
(1,5 \%)\end{array}$ & $\begin{array}{c}29 \\
(2,8 \%)\end{array}$ & $\begin{array}{c}94 \\
(4,3 \%)\end{array}$ & $\begin{array}{c}1,6 \%) \\
(1,9 \%)\end{array}$ & $\begin{array}{c}13 \% 5 \\
(3,5 \%)\end{array}$ \\
\hline Divorcés & $\begin{array}{c}2 \\
(0,1 \%)\end{array}$ & $\begin{array}{c}3 \\
(0,3 \%)\end{array}$ & $\begin{array}{c}8 \\
(0,4 \%)\end{array}$ & $\begin{array}{c}13 \\
(0,1 \%)\end{array}$ & $\begin{array}{c}21 \\
(0,2 \%)\end{array}$ & $(0,3 \%)$ \\
\hline Total & $\begin{array}{c}488 \\
(71,2 \%)\end{array}$ & $\begin{array}{c}197 \\
(28,8 \%)\end{array}$ & $\begin{array}{c}685 \\
(100 \%)\end{array}$ & $\begin{array}{c}4479 \\
(74,3 \%)\end{array}$ & $\begin{array}{c}1552 \\
(25,7 \%)\end{array}$ & $\begin{array}{c}6032 \\
(100 \%)\end{array}$ \\
\hline
\end{tabular}

